\title{
Classification of New Biomarkers of Dilated Cardiomyopathy Based on Pathogenesis-An Update
}

\author{
Muhammad Nabeel Dookhun'1,2, Yali Sun ${ }^{1}$, Huayiyang Zou' ${ }^{1}$ Xiaoqian Cao', Xinzheng Lu ${ }^{1,2^{*}}$ \\ ${ }^{1}$ Department of Cardiology, The First Affiliated Hospital, Nanjing Medical University, Jiangsu, China \\ ${ }^{2}$ Department of Cardiology, Sir Run Run Hospital Nanjing Medical University, Jiangsu, China \\ Email: *xzlu@njmu.edu.cn
}

How to cite this paper: Dookhun, M.N., Sun, Y.L., Zou, H.Y.Y., Cao, X.Q. and Lu, X.Z. (2018) Classification of New Biomarkers of Dilated Cardiomyopathy Based on Pathogenesis-An Update. Health, 10, 300-312.

https://doi.org/10.4236/health.2018.103024

Received: January 16, 2018

Accepted: March 16, 2018

Published: March 19, 2018

Copyright ( 2018 by authors and Scientific Research Publishing Inc. This work is licensed under the Creative Commons Attribution International License (CC BY 4.0).

http://creativecommons.org/licenses/by/4.0/

(c) (i) Open Access

\begin{abstract}
Dilated Cardiomyopathy (DCM) is a complex heart disease affecting the heart musculature and vasculature, involving one or several underlying pathophysiological mechanisms. Identifying potential biomarkers for dilated cardiomyopathy is a challenge owing to various aetiologies involved. Studying the biomarkers involved in DCM will ultimately give a better insight about which pathophysiological pathways are involved in the onset of the disease. Owing to its multifactorial aetiologies, response to treatment is usually poor. If we can find the exact underlying causes, a better treatment approach could be implemented. One way to obtain better insight of DCM is to study the biomarkers released. Through biomarkers, we can know which underlying mechanisms are involved. Biomarkers can provide us with clinical information such as diagnostic, prognostic, risk stratification as well as response to treatment. Underlying mechanisms such as inflammation, stress/strain, myocyte injury, matrix remodelling, oxidative stress, neurohormones involvement, among others, can contribute to the onset of DCM. Different mechanisms will yield different biomarkers. So it would be wise to classify those biomarkers involving in DCM based on their respective pathogenesis. Moreover, most importantly is to be able to make use of the information that biomarker pertains. However, specificity of those biomarkers poses a problem. One way of making these biomarkers clinically useful is to make use of a biomarker modelling score system.
\end{abstract}

\section{Keywords}

Dilated Cardiomyopathy, Biomarkers, Biomarkers Model, Biomarker Score, DCM Biomarkers, New Biomarkers 


\section{Introduction}

DCM is a common cause of heart failure, which can affect all population groups. DCM can be inherited or acquired which leads to structural and functional changes that eventually result in cardiac dysfunction and hence heart failure. Male is more affected than female with an incidence ratio of 3:1 [1] and while the exact reason still remains unclear, it can be explained by SHBG, which alters the cardiac structure and function [2]. Macroscopically, DCM is characterised by increased ventricular sizes and impaired systolic functions [3] while microscopically it is characterized by a histological triad of myocyte hypertrophy, myocyte degradation, and interstitial fibrosis [4].

DCM can be classified either as primary/idiopathic if of unknown aetiology. Gene mutations can cause familial DCM [5] [6] [7] and among the idiopathic cases, one third can be accounted to familial DCM [8] [9]. DCM can also be attributed to secondary causes including acquired conditions and diseases such as hypertension, alcohol intake, viral infections, drugs among others. The clinical consequences of DCM in paediatric populations are associated with low survival rate and poor outcome [10]. After the emergence of DCM symptoms, 50\% of DCM patients die within five years [11] [12]. In order to implement better therapeutic strategies, we need to first understand the diverse pathological mechanisms underlying DCM. One way to get a better insight about the DCM is to study the biomarkers involved in DCM (Figure 1).

\section{Biomarkers of Inflammation}

Inflammatory biomarkers are involved in the pathophysiologic mechanism of

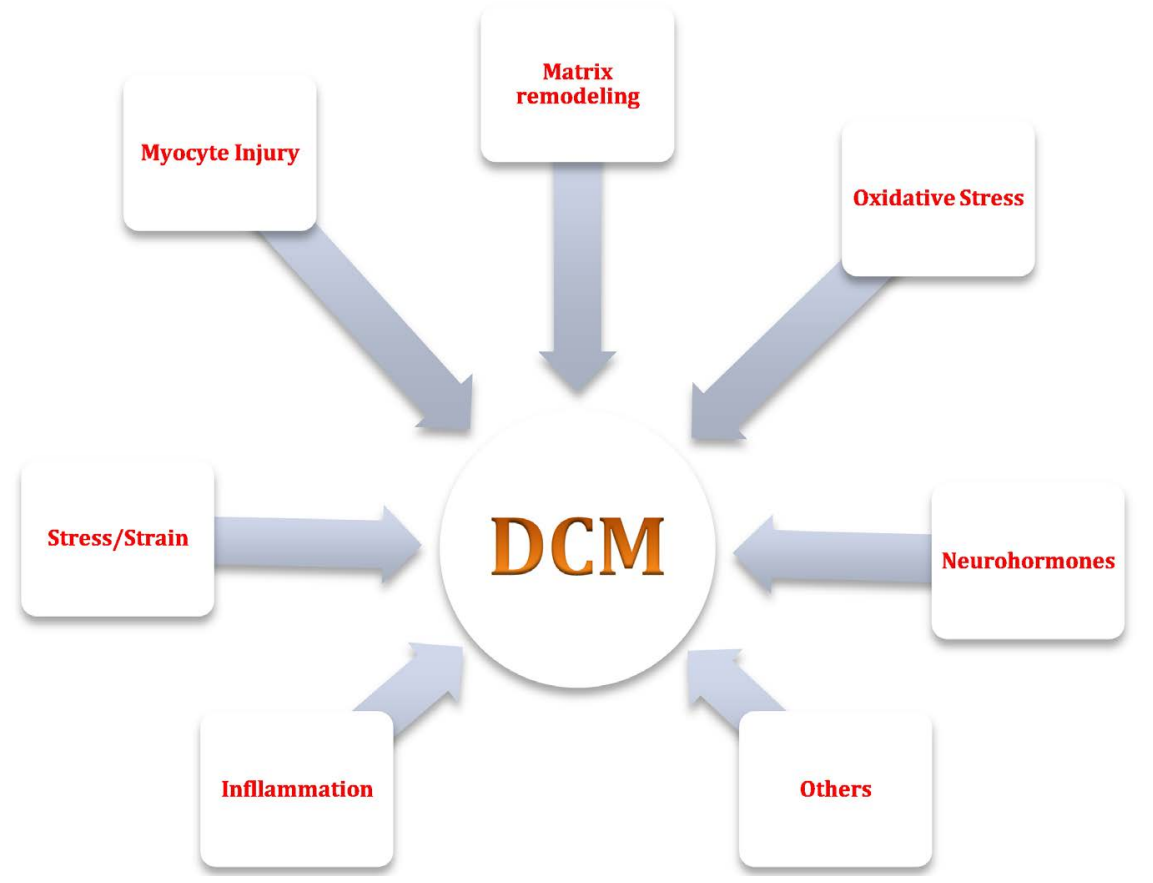

Figure 1. Classification of DCM Biomarkers based on their pathogenesis. 
heart failure and monitoring these biomarkers can provide better insight in terms of diagnosis, prognostics and outcomes of treatment [13] [14].

1) CRP (C-Reactive Protein) \& hs-CRP (Prognostic Biomarker)

CRP is an important biomarker of inflammation as well as a causative factor of endothelial dysfunction [15] [16]. Irrespective of the cause, serum level of hs-CRP is increased in CHF patients [17] [18] [19]. Based on studies, elevated hs-CRP level has been found to be a reliable prognostic biomarker of chronic heart failure [20] [21] and that CRP level can independently predict outcome of disease in DCM patients [17] [22]. Endothelial dysfunction, which can be caused by CRP, is also involved in the pathogenesis and prognosis of CHF [23]. Therefore CRP can be used as a prognostic indicator of CHF patients with DCM. The combination of hs-CRP and BNP provide a better prognostic insight in term of mortality [24]. Xiaopin Li et al. confirmed that elevated hs-CRP and NT pro-BNP have higher rate of mortality in CHF patients with DCM [25].

2) Neutrophil/Lymphocyte ratio (NLR) (Prognostic Biomarker)

Following inflammation in heart failure, leukocytes have an important implication in its pathogenesis, mainly reflecting the progression and worsening of heart failure [26] [27]. Increased mortality in HF patients is reflected by neutrophilia and lymphocytopenia [28] [29]. NLR has been shown to be an eligible biomarker for several cardiovascular diseases [30]. Moreover, NLR correlates positively with heart parameters, including LVEF and is related to severity of DCM [31]. Cardiac remodelling in DCM causes ventricular systolic dysfunction of which LVEF can be a good indicator [32]. This suggests that evaluation of chronic heart failure in DCM patients using NLR as biomarkers is a non-invasive and cheap possibility.

3) Chemerin (prognostic)

Chemerin, an adipokine secreted by adipose tissue in response to inflammatory stimulations such as TNF-alpha and IL-1B [33] [34], has been recently found to be significantly elevated in DCM patients [35]. As discussed above, LVEF is a reliable indicator of heart function in DCM patients. Previous studies have shown that positive relationship between LVEF and chemerin level does exist [35] [36]. More studies are needed to investigate whether a high level of chemerin in DCM patients is associated with recurrent adverse cardiac events.

4) Galectin-3 (Gal-3)

Gal-3, an inflammatory component, also acts a regulator of cardiac fibrosis by stimulating fibroblast proliferation that result in excessive collagen in hypertrophied heart and thus causing cardiac dysfunction [37] [38]. Cardiac fibrosis is common in DCM and its presence suggests a more serious disease condition and worse prognosis mainly due to accelerated heart dysfunction [39] [40] [41]. Vergaro et al. first reported the relationship between serum Gal-3 level and myocardial fibrosis in DCM patients [42].

\section{Biomarkers of Myocyte Stress/Strain}

1) BNP \& NT-proBNP 
BNP \& NT-proBNP are typical traditional biomarkers of heart failure used for diagnosis, screening and prognosis purposes [43]. Ventricular stresses such as pressure overload and volume expansion are responsible for the secretion of BNP [25] [44]. Increased level of BNP or NT-proBNP was found to be strong predictor of mortality and adverse cardiac events in HF and DCM [45] [46]. Owing to a longer half-life, NT-proBNP is of better clinical use than BNP [25] [47].

2) ST2/Interleukin-33

ST2, an interlukin IL-1 receptor, is known to be a biomarker of heart failure by predicting the extent of cardiac remodelling and fibrosis [48] [49]. ST2 is expressed in cardiac cells and up-regulated in response to mechanical strain and IL- $\beta$ [50]. It is considered as a superior biomarker because unlike other biomarkers such as BNP, NT-proBNP, troponin among others, is independent of age, BMI, sex, renal function or heart failure history [49]. Even being a good prognostic biomarker of heart failure no actual current report of the role of ST2 in DCM patients is available.

\section{Biomarkers of Myocyte Injury}

\section{1) High-Sensitivity Cardiac Troponin T (hs-cTnT)}

Studies have highlighted the eligibility of troponins as prognostic markers in DCM patients [51] [52]. hs-cTnT, a new generation of troponin biomarker, is now being widely used owing to its potential of detecting cardiac injury to the minimal extent. An increased level of serum hs-cTnT reflects ongoing myocardial damage [51]. Yuichi Baba et al. defined a cut-off value of $0.014 \mathrm{ng} / \mathrm{mL}$, whereby DCM patients with value less than the cut-off value tends to have reverse remodelling and those greater than the cut-off value experienced no reverse remodelling [53]. This implies hs-cTnT provides a better risk stratification in DCM patients.

2) Heart-type fatty acid binding protein (H-FABP)

$\mathrm{H}-\mathrm{FABP}$, an important fatty acid carrier protein of low molecular weight, is present abundantly in the cytoplasm [54]. Thus following cellular damage, $\mathrm{H}-\mathrm{FABP}$ is quickly released into circulation and this can be attributed to ongoing myocardial damage associated to DCM [55]. The clinical use of H-FABP and BNP combination provide better prognostic value. Elevated level of both markers suggests a worse prognosis in DCM patients [54].

3) Myosin Binding Protein-C (MyBP-C)

MyBP-C, a structural protein, is crucial for cardiac regulatory functions and possesses a $\mathrm{N}$-terminus with multiple phosphorylation sites. Phosphorylation of these PKA sites is of crucial importance reduced phosphorylation of these PKA sites has been shown in DCM patients [56]. Moreover, MyBP-C can elicit an autoimmune response, which results in production of autoantibodies. Following post-MI, proteolysis of $\mathrm{C} 0 \mathrm{C} 1$ fragment of $\mathrm{My}-\mathrm{BP}-\mathrm{C}$ elicits autoantibodies production. These autoantibodies act on other contractile cardiac protein resulting 
in autoimmune myocarditis, which ultimately progress, to DCM and HF [57]. Kasahara et al. first reported the presence of MyBP-C reactive autoantibodies in DCM patients [58]. Onset of autoimmune myocarditis and DCM has also been related to presence of cardiac protein autoantibodies [59].

\section{Biomarkers of Extracellular-Matrix Remodeling}

1) Matrix metalloproteinases (MMP) \& Tissue Inhibitors of Metalloproteinases (TIMP)

As discussed above, cardiac fibrosis occurs in DCM and remodelling of ventricles progress to HF [45]. Proliferation of fibroblast and deposition of collagen in extracellular matrix is responsible for scar tissue formation. MMPs, whose activities are markedly increased in the progression to HF, and TIMPs usually, exist in balance [60]. Inflammatory situations can disrupt the balance between MMPs and TIMPs hence causing collagen deposition and ventricular remodelling that eventually progresses to DCM and HF.

\section{Biomarkers of Oxidative Stress}

1) Myeloperoxidase (MPO)

MPO is a leukocyte heme peroxidase that has direct effect on ventricular remodelling following post infarction and is associated with neutrophil activation and inflammation [61] [62]. It has been shown that those with low LVEF $<35 \%$ on echocardiography, MPO was related to poor right ventricular dysfunction and was a good predictor of future adverse clinical outcomes [63]. MPO can not only used to predict risk in heart failure patients but also used to monitor anti-inflammatory effect in heart failure.

\section{Neurohormones Biomarkers}

1) Endothelin-1 (ET-1)

ET-1, a 21 animo acid residue with both vasoactive and mitogenic effects [64], has been reported to increase in plasma of DCM patients [65] and endothelin-1 mRNA levels to be unregulated in the heart [66]. Moreover, interestingly two polymorphisms of the endothelin type A receptor gene (EDNRA), G231A and CA363T, are differently related to the risk and mortality in idiopathic DCM patients [67] [68]. Studies have even documented that activation of endothelin is associated with a poor prognosis in DCM patients [69] [70].

\section{Others/Unclassified Biomarkers}

1) Epithelial Progenitor Cell-EPC

DCM is sometimes referred as a two hit diseases [71] for involving structural cardiac alterations and defective vascularization simultaneously [72]. EPCs are bone marrow derived cells, which are increased in response to vascular injuries through mediators such as VEGF-A and SDF-1 [73]. Studies have reported elevated EPCs level in DCM patients and have further been confirmed by Theiss et 
al. who showed raised level of CD34+ cells in DCM patients as compared to normal population [74]. This can be accounted to triggered cytokine cascade due to endothelial dysfunction in DCM [75]. There exists a relationship between increased EPC levels in DCM progression and hence EPCs can be a potential biomarker in DCM.

2) Bispherol A (BPA)

BPA is a widely used chemical mainly in plastic products and when exposed to high temperature, can mix in water and food [76] and thus can be detected in human [77]. Elevated level of BPA has been found in DCM patients as compared to healthy ones [2]. Interesting BPA can give a plausible explanation for the higher incidence of DCM in male. Several Studies showed an existing positive relation between BPA level and SHBG [78] [79]. From the study conducted by Pascual-Fgal et al., it has been demonstrated that the severity of heart failure and hence the risk of cardiac death is associated with the level of SHBG [80]. This can somehow explains the higher incidence of DCM in male population (Table 1).

Table 1. Classification of DCM biomarkers based on pathogenesis.

\section{1) Biomarkers of Inflammation}

CRP (C-Reactive Protein) and hs-CRP

Neutrophil/Lymphocyte ratio (NLR)

Chemerin

Galectin-3

TNF

Fas (APO-1)

IL $1,6,18$

2) Biomarkers of Myocyte Stress/Stretch

BNP \& NT-proBNP

ST2/IL-33

ANP

3) Biomarkers of Myocyte Injury

Cardiac Troponin I \& T

H-FABP

MyBP-C

CK MB Fraction

\section{5) Biomarkers of Oxidative Stress}

Myeloperoxidase

Oxidative low-density lipoproteins

Urinary \& plasma isoprostane

6) Neurohormones

Norepinephrine

Renin

Aldosterone

Angiotensin 2

Arginine Vasopressin

Endothelin

7) Others

Epithelial Progenitor Cells-EPC

Bispherol A

NGAL

Beta-Trace Protein (BTP)

4) Biomarkers of Extracellular-matrix remodelling

Matrix metalloproteinases

Tissue Inhibitors of Metalloproteinases

Collagen Peptides

Cardiotrophin-1 


\section{Importance of Studying \& Classifying Biomarkers in DCM}

Till now there is not a proposed guideline for the treatment and management of DCM. DCM leading to heart failure appears to have a distinct molecular pattern underlying the pathophysiology. The identification of biomarkers for dilated cardiomyopathy presents a distinct challenge due to the diverse potential etiologies. Different etiologies of DCM underline different pathological pathways that will ultimately generate different biomarkers. In this way, it would be wise to be able to classify the different biomarkers involved in DCM based on their pathogenesis. In this review, we manage to classify the newest biomarkers that could be clinically useful. Biomarkers have properties that can give us more information about the disease and may have diagnostic values or prognostic values. By classifying biomarkers involved in DCM in term of pathogenesis and their informative value, this will give us a better insight to understand the underlying etiologies involved and stage of the disease.

\section{How to Make These Biomarkers Clinically Useful}

We should highlight one of the greatest problems that arises with those biomarkers is the fact that they are not specific to DCM. Many other inflammatory conditions can cause inflammatory biomarkers to be elevated, as it is the same for other type of biomarkers. Therefore, one possible way to make these biomarkers clinically useful in DCM is to make use of a DCM-related "biomarkers modelling score system". As discussed above, those biomarkers can give us deeper insight about DCM in term of diagnosis, prognosis, risk stratification and effective treatment monitoring. So it would be interesting to be able to further classify those biomarkers in term of biomarkers of diagnosis prognosis, risk stratification or staging of DCM. Research need to be done with such application of biomarkers scoring system model for staging of DCM.

\section{Conclusion}

DCM remains a complex cardiac disease which requires more understanding. Different aetiologies account for different pathological mechanisms that can lead to the onset of DCM. Different mechanisms mean different pathways which ultimately yield different biomarkers. Many biomarkers have been found to be associated with DMC and these new emerging potential biomarkers, by studying them, can be of great clinical use. Those biomarkers are however not specific to DCM and we yet have to find a proper way to make them clinically useful. One plausible way is to make use a DCM-related biomarkers modelling score system. Research with use of such score system in DCM is yet to be explored and clinically applied.

\section{References}

[1] Merlo, M., Pivetta, A., Pinamonti, B., Stolfo, D., Zecchin, M., Barbati, G., Di Lenarda, A. and Sinagra, G. (2014) Long-Term Prognostic Impact of Therapeutic Strate- 
gies in Patients with Idiopathic Dilated Cardiomyopathy: Changing Mortality over the Last 30 Years. European Journal of Heart Failure, 16, 317-324.

https://doi.org/10.1002/ejhf.16

[2] Xiong, Q., et al. (2015) Elevated Serum Bisphenol Alevelin Patients with Dilated Cardiomyopathy. International Journal of Environmental Research and Public Health, 12, 5329-5337. https://doi.org/10.3390/ijerph120505329

[3] Lang, R.M., et al. (2005) Recommendations for Chamber Quantification: A Report from the American Society of Echocardiography's Guidelines and Standards Committee and the Chamber Quantification Writing Group, Developed in Conjunction with the European Association of Echocardiography, a Branch of the European Society of Cardiology. Journal of the American Society of Echocardiography, 18, 1440-1463. https://doi.org/10.1016/j.echo.2005.10.005

[4] Davies, M.J. and Mckenna, W.J. (1994) Dilated Cardiomyopathy: An Introduction to Pathology and Pathogenesis. Heart, 72, No. 6.

[5] McNally, E.M., Golbus, J.R. and Puckelwartz, M.J. (2013) Genetic Mutations and Mechanisms in Dilated Cardiomyopathy. Journal of Clinical Investigation, 123, 19-26. https://doi.org/10.1172/JCI62862

[6] Yuan, F., et al. (2015) A Novel NKX2-5 Loss-of-Function Mutation Predisposes to Familial Dilated Cardiomyopathy and Arrhythmias. International Journal of Molecular Medicine, 35, 478-486. https://doi.org/10.3892/ijmm.2014.2029

[7] Garciapavia, P., et al. (2013) Genetics in Dilated Cardiomyopathy. Biomarkers in Medicine, 7, 517-533. https://doi.org/10.2217/bmm.13.77

[8] Keeling, P.J., et al. (1995) Familial Dilated Cardiomyopathy in the United Kingdom. Heart, 73, 417-421. https://doi.org/10.1136/hrt.73.5.417

[9] Mestroni, L., et al. (1999) Familial Dilated Cardiomyopathy: Evidence for Genetic and Phenotypic Heterogeneity. Journal of the American College of Cardiology, 34, 181-190. https://doi.org/10.1016/S0735-1097(99)00172-2

[10] Puggia, I., Merlo, M., Barbati, G., Rowland, T.J., Stolfo, D., Gigli, M., et al. (2016) Natural History of Dilated Cardiomyopathy in Children. Journal of the American Heart Association, 5, 1-10. https://doi.org/10.1161/JAHA.116.003450

[11] Grogan, M., et al. (1995) Long-Term Outcome of Patients with Biopsy-Proved Myocarditis : Comparison with Idiopathic Dilated Cardiomyopathy. Journal of the American College of Cardiology, 26, 80-84. https://doi.org/10.1016/0735-1097(95)00148-S

[12] Michels, V.V., et al. (1992) The Frequency of Familial Dilated Cardiomyopathy in a Series of Patients with Idiopathic Dilated Cardiomyopathy. The New England Journal of Medicine, 326, 77-82. https://doi.org/10.1056/NEJM199201093260201

[13] Maeda, K., Tsutamoto, T., Wada, A., Hisanaga, T. and Kinoshita, M. (1998) Plasma Brain Natriuretic Peptide as a Biochemical Marker of High Left Ventricular End-Diastolic Pressure in Patients with Symptomatic Left Ventricular Dysfunction. American Heart Journal, 135, 825-832. https://doi.org/10.1016/S0002-8703(98)70041-9

[14] Tsutamoto, T., Wada, A., Maeda, K., Hisanaga, T., Maeda, Y., Fukai, D., et al. (1997) Attenuation of Compensation of Endogenous Cardiac Natriuretic Peptide System in Chronic Heart Failure: Prognostic Role of Plasma Brain Natriuretic Peptide Concentration in Patients with Chronic Symptomatic Left Ventricular Dysfunction. Circulation, 96, 509-516. https://doi.org/10.1161/01.CIR.96.2.509

[15] Clapp, B.R., et al. (2005) Inflammation and Endothelial Function. Circulation, 111, 1530-1536. https://doi.org/10.1161/01.CIR.0000159336.31613.31 
[16] Verma, S., et al. (2002) Endothelin Antagonism and Interleukin-6 Inhibition Attenuate the Proatherogenic Effects of C-Reactive Protein. Circulation, 105, 1890-1896. https://doi.org/10.1161/01.CIR.0000015126.83143.B4

[17] Kaneko, K., Kanda, T., Yamauchi, Y., Hasegawa, A., Iwasaki, T., Arai, M., et al. (1999) C-Reactive Protein in Dilated Cardiomyopathy. Cardiology, 91, 215-219. https://doi.org/10.1159/000006913

[18] Sato, Y., Takatsu, Y., Kataoka, K., Yamada, T., Taniguchi, R., Sasayama, S., et al. (1999) Serial Circulating Concentrations of C-Reactive Protein, Inter-Leukin (IL)-4, and IL-6 in Patients with Acute Left Heart Decompensation. Clinical Cardiology, 22, 811-813. https://doi.org/10.1002/clc.4960221211

[19] Steele, I.C., Nugent, A.M., Maguire, S., Hoper, M., Campbell, G., Halliday, M.I., et al. (1996) Cytokine Profile in Chronic Cardiac Failure. European Journal of Clinical Investigation, 26, 1018-1022. https://doi.org/10.1046/j.1365-2362.1996.2560587.x

[20] Alonso-Martinez, J.L., Llorente-Diez, B., Echegaray-Agara, M., Olaz-Preciado, F., Urbieta-Echezarreta, M. and Gonzalez-Arencibia, C. (2002) C-Reactive Protein as a Predictor of Improvement and Readmission in Heart Failure. European Journal of Heart Failure, 4, 331-336. https://doi.org/10.1016/S1388-9842(02)00021-1

[21] Yin, W.H., Chen, J.W., Jen, H.L., Chiang, M.C., Huang, W.P., Feng, A.N., et al. (2004) Independent Prognostic Value of Elevated High-Sensitivity C-Reactive Protein in Chronic Heart Failure. American Heart Journal, 147, 931-938. https://doi.org/10.1016/j.ahj.2003.11.021

[22] Chitose, I., et al. (2004) Plasma C-Reactive Protein Is an Independent Prognostic Predictor in Patients with Dilated Cardiomyopathy. Journal of Cardiac Failure, 10, S161. https://doi.org/10.1016/j.cardfail.2004.08.026

[23] Katz, S.D., et al. (2005) Vascular Endothelial Dysfunction and Mortality Risk in Patients with Chronic Heart Failure. Circulation, 111, 310-314. https://doi.org/10.1161/01.CIR.0000153349.77489.CF

[24] Ishikawa, C., et al. (2006) Prediction of Mortality by High-Sensitivity C-Reactive Protein and Brain Natriuretic Peptide in Patients with Dilated Cardiomyopathy. Circulation Journal, 70, 857-863. https://doi.org/10.1253/circj.70.857

[25] Li, X., et al. (2014) Plasma NT pro-BNP , hs-CRP and Big-ET Levels at Admission as Prognostic Markers of Survival in Hospitalized Patients with Dilated Cardiomyopathy: A Single-Center Cohort Study. BMC Cardiovascular Disorders, 14, 67. https://doi.org/10.1186/1471-2261-14-67

[26] Fu, M. (2009) Inflammation in Chronic Heart Failure: What Is Familiar, What Is Unfamiliar? European Journal of Heart Failure, 11, 111-112. https://doi.org/10.1093/eurjhf/hfn039

[27] Anker, S.D. and von Haehling, S. (2004) Inflammatory Mediators in Chronic Heart Failure: An Overview. Heart, 90, 464-470. https://doi.org/10.1136/hrt.2002.007005

[28] Arruda-Olson, A.M., Reeder, G.S., Bell, M.R., Weston, S.A. and Roger, W.L. (2009) Neutrophilia Predicts Death and Heart Failure after Myocardial Infarction: A Community-Based Study. Circulation: Cardiovascular Quality and Outcomes, 2, 656-662. https://doi.org/10.1161/CIRCOUTCOMES.108.831024

[29] Ommen, S.R., Hodge, D.O., Rodeheffer, R.J., McGregor, C.G., Thomson, S.P. and Gibbons, R.J. (1998) Predictive Power of the Relative Lymphocyte Concentration in Patients with Advanced Heart Failure. Circulation, 97, 19-22. https://doi.org/10.1161/01.CIR.97.1.19

[30] Han, Y.C., Yang, T.H., Kim, D.I., Jin, H.Y., Chung, S.R., Seo, J.S., et al. (2013) Neutrophil to Lymphocyte Ratio Predicts Long-Term Clinical Outcomes in Patients 
with ST-Segment Elevation Myocardial Infarction Undergoing Primary Percutaneous Coronary Intervention. Korean Circulation Journal, 43, 93-99. https://doi.org/10.4070/kcj.2013.43.2.93

[31] Avci, A., et al. (2014) Neutrophil/Lymphocyte Ratio Is Related to the Severity of Idiopathic Dilated Cardiomyopathy. Scandinavian Cardiovascular Journal, 48, 202-208. https://doi.org/10.3109/14017431.2014.932922

[32] Solomon, S.D., Anavekar, N., Skali, H., McMurry, J.J., Swedberg, K., Yusuf, S., et al. (2005) Influence of Ejection Fraction on Cardiovascular Outcomes in a Broad Spectrum of Heart Failure Patients. Circulation, 112, 3738-3744. https://doi.org/10.1161/CIRCULATIONAHA.105.561423

[33] Kralisch, S., et al. (2009) Interleukin-1ß Induces the Novel Adipokine Chemerin in Adipocytes in Vitro. Regulatory Peptides, 154, 102-106. https://doi.org/10.1016/j.regpep.2009.02.010

[34] Parlee, S.D., et al. (2011) Serum Chemerin Levels Vary with Time of Day and Are Modified by Obesity and Tumor Necrosis Factor- $\alpha$. Endocrinology, 151, 2590-2602. https://doi.org/10.1210/en.2009-0794

[35] Zhang, O., et al. (2015) Circulating Chemerin Levels Elevated in Dilated Cardiomyopathy Patients with Overt Heart Failure. Clinica Chimica Acta, 448, 27-32. https://doi.org/10.1016/j.cca.2015.05.018

[36] Ji, Q., et al. (2014) Chemerin Is a Novel Biomarker of Acute Coronary Syndrome But Not of Stable Angina Pectoris. Cardiovascular Diabetology, 13, 145-145. https://doi.org/10.1186/s12933-014-0145-4

[37] Sharma, U., Rhaleb, N.-E., Pokharel, S., et al. (2008) Novel Anti-Inammatory Mechanisms of N-Acetyl-Ser-Asp-Lys-Pro in Hypertension-Induced Target Organ Damage. E-American Journal of Physiology-Heart and Circulatory Physiology, 294, H1226-H1232.

[38] Sharma, U.C., Pokharel, S., van Brakel, T.J., et al. (2004) Galectin-3 Marks Activated Macro-Phages in Failure-Prone Hypertrophied Hearts and Contributes to Cardiac Dysfunction. Circulation, 110, 3121-3128. https://doi.org/10.1161/01.CIR.0000147181.65298.4D

[39] Masci, P.G., Barison, A., Aquaro, G.D., et al. (2012) Myocardial Delayed Enhancement in Paucisymptomatic Nonischemic Dilated Cardiomyopathy. International Journal of Cardiology, 157, 43-47. https://doi.org/10.1016/j.ijcard.2010.11.005

[40] Gulati, A., Jabbour, A., Ismail, T.F., et al. (2013) Association of Fibrosis with Mortality and Sudden Cardiac Death in Patients with Nonischemic Dilated Cardiomyopathy. JAMA, 309, 896-908. https://doi.org/10.1001/jama.2013.1363

[41] Masci, P.G., Doulaptsis, C., Bertella, E., et al. (2014) The Incremental Prognostic Value of Myocardial Fibrosis in Patients with Non-Ischemic Cardiomyopathy without Congestive Heart Failure. Circulation: Heart Failure, 7, 448-456. https://doi.org/10.1161/CIRCHEARTFAILURE.113.000996

[42] Vergaro, G., et al. (2015) Galectin-3 and Myocardial Fibrosis in Nonischemic Dilated Cardiomyopathy. International Journal of Cardiology, 184, 96-100. https://doi.org/10.1016/j.ijcard.2015.02.008

[43] Maisel, A.S., et al. (2002) Rapid Measurement of B-Type Natriuretic Peptide in the Emergency Diagnosis of Heart Failure. The New England Journal of Medicine, 347, 161-167. https://doi.org/10.1056/NEJMoa020233

[44] Nagagawa, O., Ogawa, Y., Itoh, H., Suga, S., Komatsu, Y. and Kishimoto, I. (1995) Rapid Transcriptional Activation and Early mRNA Turnover of BNP in Cardiocyte Hypertrophy: Evidence for BNP as an "Emergency" Cardiac Hormone against Ven- 
tricular Overload. The Journal of Clinical Investigation, 96, 1280-1287. https://doi.org/10.1172/JCI118162

[45] Braunwald, E. (2008) Biomarkers in Heart Failure. The New England Journal of Medicine, 358, 2148-2159. https://doi.org/10.1056/NEJMra0800239

[46] Tigen, K., Karaahmet, T., Cevik, C., Gurel, E., Pala, S., Mutlu, B. and Basaran, Y. (2009) Prognostic Utility of Right Ventricular Systolic Functions Assessed by Tissue Doppler Imaging in Dilated Cardiomyopathy and Its Correlation with Plasma NT-Pro-BNP Levels. Congest Heart Fail, 15, 234-239. https://doi.org/10.1111/j.1751-7133.2009.00095.x

[47] Omland, T., et al. (2007) Prognostic Value of B-Type Natriuretic Peptides in Patients with Stable Coronary Artery Disease: The PEACE Trial. Journal of the American College of Cardiology, 50, 205-214. https://doi.org/10.1016/j.jacc.2007.03.038

[48] Shah, R.V. and Januzzi Jr., J.L. (2010) ST2: A Novel Remodeling Biomarker in Acute and Chronic Heart Failure. Current Heart Failure Reports, 7, 9-14. https://doi.org/10.1007/s11897-010-0005-9

[49] Rehman, S.U., Mueller, T. and Januzzi Jr., J.L. (2008) Characteristics of the Novel Interleukin Family Biomarker ST2 in Patients with Acute Heart Failure. Journal of the American College of Cardiology, 52, 1458-1465. https://doi.org/10.1016/j.jacc.2008.07.042

[50] Weinberg, E.O., Shimpo, M., De Keulenaer, G.W., MacGillivray, C., Tominaga, S., Solomon, S.D., Rouleau, J.L. and Lee, R.T. (2002) Expression and Regulation of ST2, an Interleukin-1 Receptor Family Member, in Cardiomyocytes and Myocardial Infarction. Circulation, 106, 2961-2966. https://doi.org/10.1161/01.CIR.0000038705.69871.D9

[51] Kawahara, C., Tsutamoto, T., Nishiyama, K., et al. (2011) Prognostic Role of High-Sensitivity Cardiac Troponin T in Patients with Nonischemic Dilated Cardiomyopathy. Circulation Journal, 75, 656-661. https://doi.org/10.1253/circj.CJ-10-0837

[52] Sato, Y., Yamada, T., Taniguchi, R., et al. (2001) Persistently Increased Serum Concentrations of Cardiac Troponin $t$ in Patients with Idiopathic Dilated Cardiomyopathy Are Predictive of Adverse Outcomes. Circulation, 103, 369-374. https://doi.org/10.1161/01.CIR.103.3.369

[53] Baba, Y. et al. (2015) Clinical Significance of High-Sensitivity Cardiac Troponin T in Patients with Dilated Cardiomyopathy. International Heart Journal, 56, 309-313. https://doi.org/10.1536/ihj.14-335

[54] Schaap, F.G., van der Vusse, G.J. and Glatz, J.F. (1998) Fatty Acid-Binding Proteins in the Heart. Molecular and Cellular Biochemistry, 180, 43-51. https://doi.org/10.1023/A:1006878621126

[55] Goto, T., et al. (2003) Circulating Concentrations of Cardiac Proteins Indicate the Severity of Congestive Heart Failure. Heart, 89, 1303-1307. https://doi.org/10.1136/heart.89.11.1303

[56] Copeland, O., Sadayappan, S., Messer, A.E., Steinen, G.J., van der Velden, J. and Marston, S.B. (2010) Analysis of Cardiac Myosin Binding Protein-C Phosphorylation in Human Heart Muscle. Journal of Molecular and Cellular Cardiology, 49, 1003-1011. https://doi.org/10.1016/j.yjmcc.2010.09.007

[57] Doesch, A.O., et al. (2010) Impact of Troponin I-Autoantibodies in Chronic Dilated and Ischemic Cardiomyopathy. Basic Research in Cardiology, 106, 25-35. https://doi.org/10.1007/s00395-010-0126-Z 
[58] Kasahara, H., et al. (1994) Autoimmune Myocarditis Induced in Mice by Cardiac C-Protein. Cloning of Complementary DNA Encoding Murine Cardiac C-Protein and Partial Characterization of the Antigenic Peptides. Journal of Clinical Investigation, 94, 1026-1036. https://doi.org/10.1172/JCI117416

[59] Kaya, Z., Leib, C. and Katus, H.A. (2012) Autoantibodies in Heart Failure and Cardiac Dysfunction. Circulation Research, 110, 145-158. https://doi.org/10.1161/CIRCRESAHA.111.243360

[60] Garcia, R.A., Brown, K.L., Pavelec, R.S., Go, K.V., Covell, J.W. and Villarreal, F. (2004) Abnormal Cardiac Wall Motion and Early Matrix Metalloproteinase Activity. American Journal of Physiology-Heart and Circulatory Physiology, 288, H1080-H1087.

[61] Vasilyev, N., Williams, T., Brennan, M.L., Unzek, S., Zhou, X., Heinecke, J.W., Spitz, D.R., Topol, E.J., Hazen, S.L. and Penn, M.S. (2005) Myeloperoxidase-Generated Oxidants Modulate Left Ventricular Remodeling But Not Infarct Size after Myocardial Infarction. Circulation, 112, 2812-2820. https://doi.org/10.1161/CIRCULATIONAHA.105.542340

[62] Nicholls, S.J. and Hazen, S.L. (2005) Myeloperoxidase and Cardiovascular Disease. Arteriosclerosis, Thrombosis, and Vascular Biology, 25, 1102-1111. https://doi.org/10.1161/01.ATV.0000163262.83456.6d

[63] Tang, W.H., Tong, W., Troughton, R.W., Martin, M.G., Shrestha, K., Borowski, A., Jasper, S., Hazen, S.L. and Klein, A.L. (2007) Prognostic Value and Echocardiographic Determinants of Plasma Myeloperoxidase Levels in Chronic Heart Failure. Journal of the American College of Cardiology, 49, 2364-2370. https://doi.org/10.1016/j.jacc.2007.02.053

[64] Love, M.P. and McMurray, J.J.V. (1996) Endothelin in Chronic Heart Failure: Current Position and Future Prospects. Cardiovascular Research, 31, 665-674. https://doi.org/10.1016/S0008-6363(96)00055-7

[65] Hiroe, M., et al. (1991) Plasma Endothelin-1 Levels in Idiopathic Dilated Cardiomyopathy. American Journal of Cardiology, 68, 1114-1115. https://doi.org/10.1016/0002-9149(91)90511-I

[66] Plumpton, C., Ashby, M.J., Kuc, R.E., O’Reilly, G. and Davenport, A.P. (1996) Expression of Endothelin Peptides and mRNA in the Human Heart. Clinical Science, 90, 37-46. https://doi.org/10.1042/cs0900037

[67] Herrmann, S., et al. (2001) A Polymorphism in the Endothelin-A Receptor Gene Predicts Survival in Patients with Idiopathic Dilated Cardiomyopathy. European Heart Journal, 22, 1948-1953. https://doi.org/10.1053/euhj.2001.2626

[68] Telgmann, R., et al. (2007) The G-231A Polymorphism in the Endothelin-A Receptor Gene Is Associated with Lower Aortic Pressure in Patients with Dilated Cardiomyopathy. American Journal of Hypertension, 20, 32-37. https://doi.org/10.1016/j.amjhyper.2006.06.016

[69] Pousset, F., Isnard, R., Lechat, P., et al. (1997) Prognostic Value of Plasma Endothelin-1 in Patients with Chronic Heart Failure. European Heart Journal, 18, 254-258. https://doi.org/10.1093/oxfordjournals.eurheartj.a015228

[70] Pacher, R., Stanek, B., Hülsmann, M., et al. (1996) Prognostic Impact of Big-Endothelin Plasma Concentrations Compared with Invasive Hemodynamic Evaluation in Severe Heart Failure. Journal of the American College of Cardiology, 27, 633-641. https://doi.org/10.1016/0735-1097(95)00520-X

[71] Roura, S., et al. (2016) Circulating Endothelial Progenitor Cells: Potential Biomark- 
ers for Idiopathic Dilated Cardiomyopathy. Journal of Cardiovascular Translational Research, 9, 80-84. https://doi.org/10.1007/s12265-015-9671-z

[72] Roura, S. and Bayesgenis, A. (2009) Vascular Dysfunction in Idiopathic Dilated Cardiomyopathy. Nature Reviews Cardiology, 6, 590-598.

https://doi.org/10.1038/nrcardio.2009.130

[73] Roura, S., Gálvez-Montón, C. and Bayes-Genis, A. (2013) The Challenges for Cardiac Vascular Precursor Cell Therapy: Lessons from a Very Elusive Precursor. Journal of Vascular Research, 50, 304-323. https://doi.org/10.1159/000353294

[74] Theiss, H.D., David, R., Engelmann, M.G., Barth, A., Schotten, K., Naebauer, M., Reichart, B., et al. (2007) Circulation of CD34+ Progenitor Cell Populations in Patients with Idiopathic Dilated and Ischaemic Cardiomyopathy (DCM and ICM). European Heart Journal, 28, 1258-1264. https://doi.org/10.1093/eurheartj/ehm011

[75] Smith, C.J., et al. (1996) Reduced Gene Expression of Vascular Endothelial NO Synthase and Cyclooxygenase-1 in Heart Failure. Circulation Research, 78, 58-64. https://doi.org/10.1161/01.RES.78.1.58

[76] Michalowicz, J. (2014) Bisphenol A-Sources, Toxicity and Biotransformation. Environmental Toxicology and Pharmacology, 37, 738-758. https://doi.org/10.1016/j.etap.2014.02.003

[77] Genuis, S.J., Beesoon, S., Birkholz, D. and Lobo, R.A. (2012) Human Excretion of Bisphenol A: Blood, Urine, and Sweat (BUS) Study. Journal of Environmental and Public Health, 2012, Article ID: 185731. https://doi.org/10.1155/2012/185731

[78] Meeker, J.D., Calafat, A.M. and Hauser, R. (2010) Urinary Bisphenol A Concentrations in Relation to Serum Thyroid and Reproductive Hormone Levels in Men from an Infertility Clinic. Environmental Science \& Technology, 44, 1458-1463. https://doi.org/10.1021/es9028292

[79] Zhou, Q., et al. (2013) Serum Bisphenol-A Concentration and Sex Hormone Levels in Men. Fertility and Sterility, 100, 478-482. https://doi.org/10.1016/j.fertnstert.2013.04.017

[80] Pascualfigal, D.A., et al. (2009) Sex Hormone-Binding Globulin: A New Marker of Disease Severity and Prognosis in Men with Chronic Heart Failure. Revista Espanola de Cardiologia, 62, 1381-1387.

\section{Abbreviations}

DCM: Dilated Cardiomyopathy

LEVF: Left Ventricular Ejection Fraction

VEGF-A: vascular endothelial growth factor A

SDF-1: Stromal Cell-Derived Factor

SHBG: Sex Hormone Binding Globulin 